\title{
INIQUITY VS. AEQUITAS
}

\author{
Verfluchungen, Totenklagen, die Figur der Margaret \\ und das "Stilprinzip der Symmetrie" in Shakespeares \\ Drama , King Richard III،
}

Eine Analyse unter Berücksichtigung der modernen Ritualtheorie

Von Sandra Kluwe (Heidelberg)

Der Aufsatz betrachtet das für Shakespeares Drama ,King Richard III konstitutive „Stilprinzip der Symmetrie" (Wolfgang Clemen) im Lichte der Ritualwissenschaft. Rhetorische Figuren der Symmetrie verleihen den Szenen ritueller Verfluchung und Totenklage eine liturgischsakrale Qualität, die den durch Richard verletzten göttlichen ordo restituiert. Auch auf der tektonischen Ebene des Dramas wird das durch Richard repräsentierte Prinzip der iniquity ausbalanciert durch das Prinzip der aequitas.

The article focuses on the "stylistic principle of symmetry“ (Wolfgang Clemen) constitutive for Shakespeare's drama 'King Richard III in the light of ritual studies. Rhetorical figures of symmetry lend a sacral and liturgical dimension to the scenes of ritual cursing and lamentations for the dead, restoring the divine ordo violated by Richard. Equally, on the tectonic level of the drama, the principle of iniquity represented by Richard is balanced by the principle of aequitas.

„Fair is foul, and foul is fair $\left.{ }^{(1)}\right)$ : „Etwas ist faul ${ }^{\text {“2 }}$ ) in den meisten jener Staaten, die in Shakespeares Königsdramen zur Darstellung gelangen. Does „heaven“ „direct it "'?3) Die Perversion des ,Fairen', also Schönen, aber auch Gerechten, ins ,Faule, moralisch und politisch Hässliche, staatlich Korrupte, reißt zwar stets viele Opfer mit, wird in der Mehrheit der Königsdramen - am wenigsten in `King Lear $<$ - aber tatsächlich korrigiert. In `King Richard III ist es nicht nur die Handlung, sondern auch die schöne Magie des Klangs in den rituell-liturgisch, kultisch zelebrierend

1) William Shakespeare, Macbeth. Englisch/Deutsch. Übersetzt und hrsg. von Barbara Rojahn-Deyk, Stuttgart 1977, S. 10.

2) William Shakespeare, Hamlet, zit. nach: Shakespeare. Sämtliche Werke, hrsg. von Erich Loewenthal. Erste Abteilung: Dramatische Werke. Bd. III: Tragödien, übersetzt von August Wilhelm Schlegel und Ludwig Tieck, 5. Aufl., Heidelberg 1987, S. 495.

3) William Shakespeare, Hamlet. Englisch/Deutsch, hrsg., übers. und kommentiert von HolGer M. Klein, Bd. 1: Einführung. Text. Übersetzung. Textvarianten, Stuttgart 1984, S. 96. 
und chorisch wirkenden Szenen I/3, II/2, IV/4 und V/3, die foul in fair verwandelt und die vorübergehend zutiefst gestörte Weltordnung restituiert. Dabei scheint „heaven“ gnadenreich an der Beschwörung des Prinzips ausgleichender Gerechtigkeit, der aequitas, zu partizipieren, um Richard, als Personifikation der "Iniquity“ (III/1, V. 83, S. 112), $\left.{ }^{4}\right)$ zu bekämpfen.

Im Hinblick auf diese - nachfolgend zu plausibilisierende - These kommt einem Befund von Wolfgang Clemens ,Richard III-Kommentar entscheidende Bedeutung zu: dem Befund, dass dem ,sich im ganzen Drama in verschiedener Abwandlung wiederholende[n] Stilprinzip der Symmetrie", das typisch für Shakespeares Frühstil sei, „etwas Absichtsvolles, Beispielhaftes“ eigne. ${ }^{5}$ ) In der Tat lässt kaum ein frühes Drama Shakespeares Goethes Ausruf „Natur! Natur! Nichts so sehr Natur wie Shakespeares Menschen" ${ }^{\text {") }}$ ) so unzutreffend erscheinen wie 'King Richard III, und kaum eine Figur dieses Dramas ist so dezidiert künstlich, besser gesagt: kunstvoll in ihrer Machart und ihrem Wirkungsprinzip wie die Figur der Margaret. $\left.{ }^{7}\right)$

Zwei spezifizierende Fragen sind im Anschluss an Clemens Befund zu stellen: (1) Wie konkretisiert Shakespeare das für 'King Richard III so typische „Stilprinzip der Symmetrie"? Diese Frage zielt a) auf tektonische und b) auf rhetorische Figuren wie z.B. repetitio, Anapher, Epipher, Parallelismus ab. (2) Worin liegt der Kern der künstlerischen Intention von 'King Richard III, sofern sich diese in repetitio- und Symmetrie-Figuren als zentralem Strukturprinzip des Dramas manifestiert? - Die Antwort sei an dieser Stelle thesenhaft antizipiert.

\section{Ritus und ordo}

Festzustellen ist zunächst, dass dem tektonisch-rhetorischen Symmetrieprinzip etwas Liturgisch-Sakrales zukommt. Das ästhetische Moment der Symmetrie eröffnet den Darstellungsraum des Rituellen, der dem Ritus des ordo missae, gleichzeitig aber einem magisch-archaischen Ritus entspricht.

4) Hier und im Folgenden wird mit Seitenangaben in Klammern zitiert nach der Ausgabe: William Shakespeare, King Richard III/König Richard III. Englisch/Deutsch, übersetzt und hrsg. von Herbert Geisen, Stuttgart 1978.

5) Wolfgang Clemen, Kommentar zu Shakespeares Richard III. Interpretation eines Dramas, Göttingen 1969, S. 16. - Marie-Hélène Besnault und Michel Bitot, Historical legacy and fiction. The poetical reinvention of King Richard III, in: The Cambridge companion to Shakespeare's history plays, hrsg. von Michael Hattaway, Cambridge 2002, S. 106-125, hier: S. 107: „Richard III can be seen as the ultimate exemplum, a political emblem for human degradation, set against the idealised regeneration." Im - wiewohl berechtigten - Verweis auf die Nähe zur exemplum-Literatur erschöpft sich die von Clemen konstatierte Ausrichtung der Darstellungsabsicht auf das Beispielhafte allerdings nicht.

$\left.{ }^{6}\right)$ Johann Wolfgang Goethe, „Zum Shakespears Tag“ (1771). Zit. nach: Ders., Sämtliche Werke, Frankfurter Ausgabe in 40 Bänden, I. Abteilung, Bd. 18: Ästhetische Schriften 1771-1805, hrsg. von Friedmar Apel, Frankfurt/M. 1998, S. 9-12, hier: S. 11.

$\left.{ }^{7}\right)$ Das Shakespeare-Handbuch (Die Zeit, der Mensch, das Werk, die Nachwelt, hrsg. von InA Schabert, 5. Aufl., Stuttgart 2009, S. 346) attestiert dem Drama „rituelle Künstlichkeit“. 
Im Deutschen ist das Wort ,Ritus' seit dem 17. Jahrhundert belegt und bezieht sich zunächst auf einen religiösen Gebrauch, ${ }^{8}$ ) beispielsweise den „Abendmahlsritus.“9) Wer rite, d. h. dem tradierten Gebrauch gemäß verfährt, wird der dem jeweiligen Ritus eingeschriebenen „Handlungsnorm“ gerecht und erreicht das angestrebte soziale Ziel. ${ }^{10}$ ) Ziel ist die Wiederherstellung einer vorübergehend gestörten Weltordnung, die durch die Performanz des jeweiligen ,Rituals' als Normativität angemahnt und mit magischer Kraft beschworen wird: Rituale, so die zentrale Annahme zeitgenössischer Ritualforschung, die in vorliegenden Ausführungen herangezogen werden soll, ,vergegenwärtigen eine als ursprünglich gesetzte Anfangsnormativität einer Gemeinschaft ". ${ }^{11}$ ) Die Performanz des Rituals „,setzt die Invarianz und Dauer der Anfangsnormativität als Prätext voraus, dessen

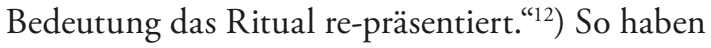

Rituale einen eminent normativen Charakter: Sie fordern eine Gemeinschaftlichkeit im gemeinsamen Handeln, sie gebieten die Bedingungen der Möglichkeit einer (transparenten) Kommunikation aller Beteiligten, sie bilden ein moralisches Apriori, das eine unhinterfragte soziale Gültigkeit beansprucht. Diesen normativen Charakter gewinnen Rituale durch die impliziten Regeln rituellen Handelns, die nicht in Frage gestellt werden dürfen. $\left.{ }^{13}\right)$

„Die Normativitätsvermittlung verschafft Ritualen einen Bezug zur Macht. ${ }^{\text {"14 })}$ Aufgrund dieser - ursprünglich und kontinuierlich religiös konnotierten - Normativität zieht der Ritus auch eine Grenze zwischen dem Heiligen und dem Profanen: Wer sich diesseits des Ritus bewegt, achtet den Raum des Sakralen; wer den Ritus stört, gefährdet den Raum des Sakralen und droht zu profanisieren, was einer Gesellschaft - im engeren oder erweiterten Sinne - , heilig' ist.

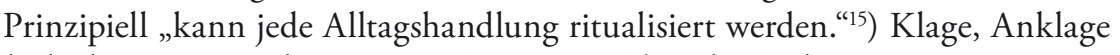
und Fluch, wie sie in den Szenen I/3, II/2, IV/4 und V/3 des Dramas 'King Richard III begegnen, sind hierfür jedoch in besonderem Maße disponiert, da sie „neuralgische Punkte des öffentlichen und des individuellen Lebens" betreffen: ${ }^{16}$ )

${ }^{8}$ ) Burckhard Dücker, Rituale: Formen - Funktionen - Geschichte. Eine Einführung in die Ritualwissenschaft, Stuttgart und Weimar 2007, S. 14.

9) Ebenda, S. 15.

$\left.{ }^{10}\right)$ Ebenda.

${ }^{11}$ ) Metzler Lexikon Literatur- und Kulturtheorie. Ansätze - Personen - Grundbegriffe, hrsg. von Ansgar Nünning, 4. Aufl., Stuttgart und Weimar 2008, S. 630.

12) Ebenda, S. 631.

13) Christoph Wulf und Jörg Zirfas, Performativität, Ritual und Gemeinschaft. Ein Beitrag aus erziehungswissenschaftlicher Sicht, in: Ritualdynamik. Kulturübergreifende Studien zur Theorie und Geschichte rituellen Handelns, hrsg. von Dietrich Hardt und Gerrit Jasper Schenk, Heidelberg 2004, S. 73-93, hier: S. 89.

$\left.{ }^{14}\right)$ Metzler Lexikon Literatur- und Kulturtheorie (zit. Anm. 11), S. 630.

15) DüCKer, Rituale (zit. Anm. 8), S. 1.

16) Ebenda. 
die Verletzung des Rechts auf Leben und Regentschaft durch Mord an Königen und Thronfolgern. ${ }^{17}$ )

Auf der formalen Ebene sind rituell wirkende Texte und Sprechakte ,expressiv stilisiert' und repetitiv-gleichförmig. ${ }^{18}$ ) Wolfgang Braungart gibt folgende Definition des Rituals, die sich auch auf literarische Rituale bzw. literarische Texte mit rituellem Charakter anwenden lässt: „Ein Ritual wird meines Erachtens konstituiert: a) durch die Wiederholung einer Handlung; b) durch Festlichkeit und Feierlichkeit; c) durch Selbstbezüglichkeit; d) durch Akteure und Zuschauer, die sich der Bedeutsamkeit des Rituals bewußt sind; e) durch eine ästhetisch-symbolische Ausgestaltung, die das Ritual heraushebt und unterscheidet", durch Repetivität einer „Handlung oder Handlungssequenz“, die den Alltag unterbricht, sowie durch „Formbewußtsein“ ${ }^{19}$ ) Zum „Formbewußtsein“ gehören Aspekte der multimodalen Performanz mit ihren prosodischen Merkmalen Sprachmelodie, Lautstärke, Tempo, Tonfall, Pausen (bis hin zu Mimik und Gestik), also der „Vortragsart ${ }^{\prime 2}{ }^{20}$ ) Es sind Vortragsarten wie „Rezitation, Lesung, Gesang, Anrufung“, die dem jeweiligen Text den Charakter der „rituellen Performanz“ geben. ${ }^{21}$ ) Rezitativische, chorisch-lyrische Elemente sowie Annäherungen an einen polyphonen Wechselgesang ${ }^{22}$ ) sind in den erwähnten Szenen I/3, II/2, IV/4 und V/3 des Dramas 'King Richard III von eminenter Bedeutung.

Ein solcherart rituell-sakrales, der liturgischen Tendenz des morality play angenähertes Sprechen evoziert eine spezifisch mittelalterlich konnotierte „Anfangsnormativität", die als eminent fair - im Doppelsinn von Schönheit und Gerechtigkeit - anzusehen ist: Die Beschwörung des verlorenen und wiederherzustellenden Zustands, des ordo, ${ }^{23}$ ) der Gerechtigkeit (aequitas), die von Richards

${ }^{17}$ ) Nach E.M.W. Tillyard (Shakespeare’s history plays, London 1956, S. 199) ist „Richard III" ,strong in the ritual element".

18) Rainer E. Wiedenmann, Ritual und Sinntransformation. Ein Beitrag zur Semiotik soziokultureller Interpenetrationsprozesse, Berlin 1991, S. 13.

19) Wolfgang Braungart, Ritual und Literatur. Literaturtheoretische Überlegungen im Blick auf Stefan George, in: Sprache und Literatur in Wissenschaft und Unterricht 23 (1992), H. 69, S. 2-31, hier: S. 4 bzw. 6.

20) Burckhard Dücker und Hubert Roeder, Einleitung, in: Text und Ritual. Kulturwissenschaftliche Essays und Analysen von Sesostris bis Dada, hrsg. von BURCKHARD DücKer und Hubert Roeder, Heidelberg 2005, S. 9-15, hier: S. 9.

${ }^{21}$ ) Ebenda.

22) Vgl. Clemen, Kommentar (zit. Anm. 5), S. 251.

23) Trotz des Vorbehalts, dass „die Elisabethaner kein gemeinsames Weltbild“ hatten, dieses vielmehr durch Inkongruenzen im Übergang zwischen dem stabilen ordo-Denken des Mittelalters und dem dynamischeren Weltbild der Renaissance geprägt war (vgl. ULRICH Suerbaum, Shakespeares Dramen, 2. Aufl., Tübingen und Basel 2001, S. 85), wird man folgende communis opinio festhalten können: „Die Elisabethaner fassen das Weltganze als frame of order, als Bauwerk der Ordnung, auf. [...] Man streitet über die Grenzen ihrer Erkennbarkeit und über den Grad ihres Zerfalls, nicht aber darüber, daß es im Prinzip eine universale Ordnung gibt, in der alle gegenwärtigen und vergangenen Phänomene ihren Platz haben und die sowohl materielle als auch geistige Wesenheiten umfaßt. [...] 
Intrigen und Mordanschlägen gestört wurden, gelingt mit genuin ästhetischen Mitteln, doch sind diese nicht Selbstzweck, sondern stehen im Dienste einer primär religiös fundierten Kongruenz von Form und Inhalt. Um diese Sinngebung des Strukturprinzips der Symmetrie zu indizieren, soll im Rahmen vorliegender Ausführungen das Wort aequitas Verwendung finden, das, als Etymon von Iniquity, den Kontrapunkt des Bösen, kurz: das gegen Richard streitende Prinzip des Guten zur Darstellung bringt. Richard nämlich präsentiert sich dem Publikum im dritten Akt als ein Nachfahr des vice, Personifikation teuflischer Laster im christlichen morality play: $\left.{ }^{24}\right)$,[Aside.] Thus, like the formal Vice, Iniquity, | I moralize two meanings in one word" (III/1, V. 83, S. 112).

Iniquity, in der Bedeutung von „Ungerechtigkeit, Schlechtigkeit, Schändlichkeit", ist das moralische Äquivalent zur Asymmetrie. Symmetrie hingegen, als schönes Ebenmaß, Ausgewogenheit und Harmonie der Komposition, kosmos, als das rechte Maß, der goldene Schnitt usw. steht in der Kunst des Mittelalters und der Neuzeit zumeist für den göttlichen ordo, innerhalb dessen das Schöne ontotheologisch fundiert ist, da Schönheit, Wohlproportioniertheit und Symmetrie als Zeichen Gottes gelten. So übernimmt Thomas von Aquin von Pseudodionysius den „Aspekt der Proportion (Harmonie), der [...] im Kontext der Trinitätslehre durch den Aspekt der Vollkommenheit (perfectio, integritas)" ergänzt wird. ${ }^{25}$ )

In 'King Richard III muss das Augenmerk, wenn vom Begriff des ,Schönen die Rede ist, zudem auf die eingangs erwähnte doppelte Bedeutung des Wortes fair gelegt werden. Etymon von fair - in dieser doppelten Bedeutung „schön“ und ,gerecht" " ist das altsächsisch-althochdeutsche Wort fagar. Zu althochdeutsch fagar

Die Einheit des Universums stammt aus Gott. Seiner einen Schöpfungsintention ordnen sich das Ganze und alle Teile unter. Er erhält sein Werk und gibt dem Menschen auf, die Ordnung zu erkennen und sich ihrer zu erfreuen. An die Ordnung der Welt zu denken, hat daher stets religiöse Implikationen" (ebenda, S. 87). - Vgl. im Zusammenhang mit dem ordo-Weltbild des Mittelalters Dietrich Hardt, Rituale, Texte, Diskurse. Eine formtheoretische Betrachtung, in: Text und Ritual. Kulturwissenschaftliche Essays und Analysen von Sesostris bis Dada, hrsg. von Burckhard Dücker und Hubert Roeder, Heidelberg 2005, S. 19-48, hier: S. 19: „,Ritual' verweist mit „den Merkmalen des ,Richtens' und ,Rechtens' (rite) [...] fraglos auf die Vorstellungen einer Ordnungssemantik." Hardt (ebenda, S. 33): „Es ist die auf Kontinuität bauende Formkraft des Rituellen, die [...] Ordnung an der Schwelle zur Ordnungs(zer)störung garantiert“. - Vgl. ferner Christiane Wiтtнöfт, Ritual und Text. Formen symbolischer Kommunikation in der Historiographie und Literatur des Spätmittelalters, Darmstadt 2004, S. 1: „In der Präsenzkultur des Mittelalters sind Rituale neben anderen Formen symbolischer Kommunikation ein tragender Pfeiler der politischen Ordnung."

${ }^{24)}$ Nach Tillyard (Shakespeare's history plays, zit. Anm. 17, S. 200) besteht „much evidence that Shakespeare wrote his tetralogy deliberately and academically and that he was deeply influenced by the Morality tradition with its medieaval passion for equivalences".

${ }^{25}$ ) Josef Wohlmuth, ,Schönheit/Herrlichkeit“, in: Neues Handbuch theologischer Grundbegriffe, erweiterte Neuausgabe, hrsg. von Peter Eicher, München 1991, Bd. 5, S. 17-25. Vgl. hierzu außerdem Umberto Eco, Kunst und Schönheit im Mittelalter, aus dem Italienischen von Günter Memmert, München und Wien 1991. 
(,prächtig, blendend“) gehört das Substantiv fagari („Glanz, Herrlichkeit“), ${ }^{26}$ ) das Äquivalent zu hebräisch kabod Jahwe ${ }^{27}$ ) und lateinisch splendor/gloria Dei.

In der theologia gloriae wird die „Herrlichkeit des Herrn“ als „splendor Dei, als Glanz oder Schein der Herrlichkeit Gottes" ästhetisiert. ${ }^{28}$ ) Wenn das solcherart konzipierte Schöne Bestand hat, wenn ordo und kosmos Vorschein göttlichen Glanzes sind, stehen Himmel und Erde, das göttlich Gute und das menschlich Verführbare, in einem ausbalancierten Verhältnis zueinander und erweist sich auch die menschliche Seele als, schön'.

Aufgabe ästhetischer Erziehung im Sinne der Didaxe eines morality play wäre es demnach, die Seele, trotz ihrer Berührung mit dem kontaminierenden vice, ,schön', also ästhetisch geläutert aus der Rezeption des Dramas treten zu lassen. Schlicht formuliert: Der moral sense - als ein sense of beauty (Hutcheson) - sollte be/friede/t aus dem Theater gehen. Auch aus gestaltpsychologischen Gründen. Dies, so die auf Anhieb vielleicht eher verwunderliche These, ist in 'Richard III der Fall, sofern die Aufführung und das Publikum die Szenen I/3, II/2, IV/4 und $\mathrm{V} / 3$ in das Gestaltkonzept des Ganzens integrieren und entsprechend der Absicht des Autors auf sich wirken lassen.

\section{Margaret: Grundzüge der Figurengestaltung}

Die Figur der Margaret ist innerhalb eines solchen Gestaltkonzepts als „balance of the play ${ }^{(29)}$ aufzufassen. Ihre selbstdeklarierte Funktion, ,the repetition of what thou hast marr'd" (I/3, V. 165, S. 46), die Wiederholung dessen, was Richard „angerichtet" hat (S. 47), „Wiederholung des, was du zerstört" , ${ }^{30}$ ) wird auf der rhetorischen Ebene durch Figuren der Wiederholung und auf der tektonischen Ebene durch das bereits skizzierte Prinzip der aequitas als Symmetrie umgesetzt. Die Symmetrie einzelner Szenen, darunter der Werbungs- und Fluchszenen, profiliert das von Margaret berufene rise-and-fall-Schema: „They that stand high have many blasts to shake them, | And if they fall, they dash themselves to pieces" (I/3, V. 260, S. 52). Gemäß dem Talions-Gesetz kommen die zwei Schalen derselben Waage der Gerechtigkeit durch die zweite der jeweils symmetrisch korrespondierenden Szenen zum Ausgleich.

${ }^{26)}$ Rudolf Schützeichel, Althochdeutsches Wörterbuch, Tübingen 1995, S. 127.

${ }^{27}$ ) Zur kabod Jahwe vgl. Thorleif Boman, Das hebräische Denken im Vergleich mit dem griechischen, 5. Aufl., Göttingen 1968, S. 73.

${ }^{28}$ ) Lexikon der Ästhetik, hrsg. von Wolfhart Henckmann und Konrad Lotter, München 1992, S. 215

29) Besnault/Biot, Historical legacy (zit. Anm. 5), S. 118. - Tillyard (Shakespeare's history plays, zit. Anm. 17, S. 199) streicht die „formal balance“ von $\curvearrowright$ Richard III heraus.

$\left.{ }^{30}\right)$ William Shakespeare, König Richard III, zit. nach: Shakespeare, Sämtliche Werke, hrsg. von Erich Loewenthal. Erste Abteilung: Dramatische Werke, Bd. II: Historien, übersetzt von August Wilhelm Schlegel und Ludwig Tieck, 5. Aufl., Heidelberg 1987, S. 694. 
Durch die dreifache Umsetzung des Wiederholungsprinzips (inhaltlich, tektonisch, rhetorisch) erweist sich Shakespeares 'King Richard III als ein eminent rituelles Drama. Wenn nämlich „von der Wiederholung oder Repetivität rituellen Handelns gesprochen wird, so ist damit gemeint, dass eine rituelle Handlung überhaupt erst dann vorliegt, wenn sie das Merkmal des Wiedererkennens erfüllt. “'31) Und es ist die Figur der Margaret, die für „repetition“ einsteht, die in ritueller Form wieder/holt und zum Ausgleich bringt, was Richard, die IniquityFigur, zerstörte und in Vergessenheit geraten lassen wollte. Auf diese Weise hebt Shakespeare den drameninternen ,Spielleiter' Richard genau dadurch auf, dass er dessen einseitige Sicht auf das Geschehen überschreitet und ausgleicht: durch Margaret. Ausgerechnet Margaret, die als historische Margaret von Anjou den Rosenkrieg auslöste und als fiktive Margaret, zumal in der Henriade, Richards counterpart eines weiblichen villain abgibt, sollte das schöne Prinzip der aequitas personifizieren?

Weder die historische Margaret von Anjou noch die fiktive Margaret Shakespeares ist eine Immaculata; sie hat den Mord an Rutland auf sich geladen und bleibt, wegen des Verlusts ihres Ehemanns und Sohns, Zeit ihres Lebens „hungry for revenge" (IV/4, V. 61, S. 188). ${ }^{32}$ ) Über den Tod von Clarence und das Leid seiner Mutter, der Herzogin von York, triumphiert sie, obwohl Clarence zu den Mordopfern Richards gehört. Trägt Clarence doch Schuld am Tod ihres Edward (IV/4, V. 55-58, S. 186):

O! upright, just, and true-disposing God,

How do I thank thee that this carnal cur

Preys on the issue of his mother's body,

And makes her pew-fellow with others' moan.

O redlich ordnender, gerechter Gott!

Wie dank' ich dir, daß dieser Metzgerhund

In seiner Mutter Leibesfrüchten schwelgt

Und macht sie zur Gesellin fremder Klagen! ${ }^{33}$ )

In `König Heinrich der Sechste - Dritter Teik wird Margaret von York als „Tigerherz, in Weiberhaut gesteckt“ (I/4) ${ }^{34}$ ) und als „Amazonen-Trulle“ beschimpft. ${ }^{35}$ ) „Weiber“, so der Verbal-Angriff auf die unweiblich starke Margaret, seien „sanft,

31) DüCKer, Rituale (zit. Anm. 8), S. 42.

32) Vgl. Warren Chernaik, The Cambridge Introduction to Shakespeare's History Plays, Cambridge 2007, S. 62: „The baleful Queen Margaret is a figure in the Senecan tradition, who could as easily have been given the allegorical name Revenge, framing the action of the play (as in Kid's Spanish Tragedy) by foreshadowing future reversals of fortune and calling down curses on the heads of her enemies. Again and again, her prophecies come true".

33) William Shakespeare, König Richard III (zit. Anm. 30), S. 749.

$34)$ Ebenda, S. 609.

${ }^{35}$ ) Ebenda, S. 608. 
mild, mitleidsvoll und biegsam“; Margaret hingegen „starr, verstockt, rauh, kieselhart, gefühllos. ${ }^{\text {(36) }}$

Margaret, kein Zweifel, ist nicht nur eine Königin von unzerstörbarem Stolz, sondern verkörpert darüber hinaus ein Stück weiblicher Bosheit - nicht jedoch als weibliches Pendant zum schalkhaften vice, da sie eine durch und durch tragödienhaft konzipierte Figur ist. Eingebettet in die Schar von Richards Opfern, die Margaret, obschon von diesen zunächst verkannt, anführt, wird Margarets eigene Mörderinnen-Natur allerdings im Sinne berechtigter Racheansprüche legitimiert: Wie die meisten Flüche, dient auch ihr - durch ungesühntes Leid und Verhütung weiteren Unheils gerechtfertigter - Fluch der „Herabrufung göttlicher Strafe“.37) So bleibt ein ,Höllenrest zu tragen peinlich', ein elementarer Rest alttestamentarischer Rache-Energie, der mythologisch über weibliche Schicksalsgottheiten wie die Nemesis vorgeprägt ist. ${ }^{38}$ ) Dennoch verhält es sich mit Margaret ähnlich wie mit Schillers Maria Stuart: Gerade die Schuldfähigkeit verleiht dieser Figur eine moralische Position mit Schwergewicht. ${ }^{39}$ ) Auf der Waagschale der Sühne und des Einforderns göttlicher Maßstäbe wiegt das Leid Margarets schwer genug, um nicht etwa das Zünglein an der Waage der Gerechtigkeit zu sein, nein: Vielmehr personifiziert Margaret die Waage der Gerechtigkeit selbst.

Auch aus inhaltlich-didaktischen und keineswegs nur aus tektonischen Gründen gilt somit: „Even if her [Margaret's] action appears to be mainly verbal and is concentrated in two scenes, Margaret's dismissal from performances utterly destroys the balance of the play. ${ }^{(40}$ )

Diese kunstvoll arrangierte Schlüsselrolle Margarets, die sich auch, aber keineswegs nur aus ihrer Schlüsselrolle in der Tetralogie 'König Heinrich der Sechste, Teil I-IIK und 'King Richard III ableiten lässt, ${ }^{41}$ ) rührt einerseits stets aus der

36) Ebenda, S. 609.

$\left.{ }^{37}\right)$ Dtv-Lexikon in 20 Bänden [erarb. nach Unterlagen der Lexikon-Redaktion des Verlages F. A. Brockhaus, Mannheim], München 1995, Bd. 6, S. 16.

38) Shakespeare-Handbuch (zit. Anm. 7), S. 346: „Richard III ist strukturell geprägt von einer Dialektik der Erinnerung und des Vergessens sowie von Mustern der Präfigurierung und Wiederholung. Kontinuität ergibt sich durch die Nemesis, die die Figuren in einen unentrinnbaren Kreislauf von Schuld und Sühne stellt. Diesem antiken, an Senecas Tragödien orientierten Bezugsrahmen entsprechen rituelle Künstlichkeit und ein melodramatischer Charakter. Margarets wichtige Rolle als Antagonistin Richards wird häufig übersehen. Sie tritt als Beobachterin und Kommentatorin auf sowie als personifizierte Vorsehung".

39) Besnault/Biot, Historical legacy (zit. Anm. 5), S. 117: „In Richard III [...] Shakespeare makes Margaret the ,counterweight to the mesmerising theatricality of Richard" [Zitat aus Robert Smallwood, Shakespeare's Use of History, in: The Cambridge Companion to Shakespeare Studies, hrsg. von Stanley Wells, Cambridge u. a., S. 143-162, hier: S. 155].

${ }^{40)}$ Besnault/Biot, Historical legacy (zit. Anm. 5), S. 118.

${ }^{41}$ Ebenda, S. 119: „In defiance of historical accuracy, she [Margaret] is made to appear in all four plays of the tetralogy, which makes her instrumental in linking the plays together, but which also gives the sense that she is a figure out of time, or an embodiment of Time, an ageless figure of moral nemesis“". Chernaik, The Cambridge Introduction (zit. Anm. 32), 
Konfiguration der dramatis personae, andererseits aus Margarets Isolation. Es kommt ihr eine Scharnierfunktion zu, die jedes Mal greift, wenn sich Richards Opfer der Flüche Margarets er/inner/n, d.h. dieser Flüche sub specie aeternitatis innewerden. „Sieht man Richard als ,Spielleiter, so muß man auch Margaret in solcher Funktion betrachten, denn die Flüche, die sie anfangs ausspricht (I,iii), verwirklichen sich geradezu rituell, wobei sich jede Figur bei ihrem Untergang der Worte Margarets erinnert. Gegen diese deterministische Logik lehnt sich der Politiker Richard vergeblich auf." ${ }^{(42}$ )

Grey. Now Margaret's curse is fall'n upon our heads,

When she exclaim'd on Hastings, you, and I,

For standing by when Richard stabb'd her son.

Riv. Then curs'd she Richard, then curs'd she Buckingham,

Then curs'd she Hastings: O! remember, God,

To hear her prayer for them, as now for us; (III/3, V. 14 ff., S. 130)

Hast. [...] O Margaret, Margaret! now thy heavy curse | Is lighted on poor Hastings' wretched head. (III/4, V. 91f., S. 136-138)

Buck. [...] Thus Margaret's curse falls heavy on my neck:

'When he', quoth she, 'shall split thy heart with sorrow,

Remember Margaret was a prophetess.'

Come, lead me, officers, to the block of shame:

Wrong hath but wrong, and blame the due of blame. (V/1, V. 3-29, S. 222)

Vergleichbar den guten Stimmen der Moralität versucht Margaret schon im ersten Akt des Dramas, die von Richard Verführten vom Laster abzuziehen. ${ }^{43}$ ) Die von ihrem Fluch Getroffenen sterben im reuigen Bewusstsein ihrer Schuld und ihrer persönlichen Verantwortung für die blutige Tyrannei Richards und das Leid Englands: „Hast. Woe, woe for England! not a whit for me; [...] O bloody Richard! miserable England!“ (III/4, V. 79 u. 102, S. 136 u. 138).

In ihren letzten Auftritten, in denen sie den Fluch-Ritus performativ nach/vollziehen, erlangen die von Margaret Verfluchten darüber hinaus eine letzte Würde,

S. 61: „One of her [Margaret's] functions is to serve as a reminder of past events, calling the attention of the theatrical audience to the earlier plays in the tetralogy". Ebenda, S. 62: Queen Margaret sei eine Nemesis-Figur, „whose ritual incantations give her an unearthly, supernatural quality."

${ }^{42}$ ) Shakespeare-Handbuch (zit. Anm. 7), S. 346

$\left.{ }^{43}\right)$ Shakespeare, König Richard III (zit. Anm. 30), S. 697: „Margareta. [...] | O Buckingham, weich' aus dem Hunde dort! | Sieh, wann er schmeichelt, beißt er; wann er beißt, | So macht sein gift'ger Zahn zum Tode wund. | Hab' nichts mit ihm zu schaffen, weich' ihm aus! | Tod, Sünd' und Hölle haben ihn gezeichnet, | Und ihre Diener all umgeben ihn. [...] Wie? Höhnst du mich für meinen treuen Rat | Und hegst den Teufel da, vor dem ich warne? | O denke des auf einen andern Tag, | Wenn er dein Herz mit Gram zerreißt, und sage: | Die arme Margareta war Prophetin." 
die selbst den schuldigsten Bündnispartner Richards, Buckingham, als im christlichen Sinne erlösungsfähig erweist und ihm zumindest für seine Haltung im Angesicht des Todes den Respekt des Publikums sichert.

Nach E. M. W. Tillyard besteht die Intention der Tetralogie darin, „the working out of God's plan to restore England“ dramatisch zu entwickeln. ${ }^{44}$ ) Die Erfüllung der Flüche repräsentiert demgemäß ein Weltbild, innerhalb dessen alle Sünden von einem gerechten Gott gestraft werden im Sinne eines Tun-Ergehen-Zusammenhangs, ${ }^{45}$ ) sodass Gott sich nicht nur als Herr der (Heils-)Geschichte, sondern auch und gerade der englischen Realgeschichte erweist: „the theme of order and chaos, of proper political degree and civil war, of crime and punishment, of God's mercy finally tempering his justice, of the belief that such had been God's way with England.“" $\left.{ }^{46}\right)$ Für Tillyard ist `King Richard III ‘ demnach „a very religious play“, wobei er betont, vom Drama und nicht von Shakespeare zu sprechen. ${ }^{47}$ )

Margarets Auftritte, zumal der Auftritt der zwei mit ihr zusammen klagenden Frauen, sind „vom hohen Stil geprägt “. ${ }^{48}$ ) Dieser hohe Stil dient im morality play der göttlichen Legitimierung entsprechender Figuren. Das Shakespeare-Handbuch kann Margaret vor diesem Hintergrund sogar als „personifizierte Vorsehung“ deuten..$^{49}$ )

Tatsächlich wird ein Stück Metanoia und ein Stück Koinonie des Wahren, Schönen und Guten dramatisch gestaltet, indem Richards Opfer und Mittäter oder Mitwisser im Moment der Anagnorisis nicht nur ihre Hamartia erkennen, sondern sich darüber hinaus der Prophezeiungen Margarets auf symmetrisch,schöne Weise erinnern und hierdurch offen für die ,gute Kraft des von Margaret im Drama immer wieder angerufenen, wenn auch ansonsten weitgehend absenten Gottes des Christentums sind.

Wenn nun die Idee des Dramas in der aequitas als Kontrapunkt des iniquityPrinzips besteht, dann gelingt Shakespeare in `King Richard III das „sinnliche Scheinen“ dieser Idee (des Guten), also ein im Sinne Hegels ,schönes' Drama - und dies keineswegs trotz, sondern wegen der Monstrosität des Inhalts, der nach ästhetischer Bewältigung verlangt, um das moralische Bewusstsein nicht zu verletzen. ${ }^{50}$ ) Wenn ferner, nach dem Wertesystem des Mittelalters, gilt: „Adel ist [idealiter] per se soziale Schönheit“, ${ }^{\text {51) }}$ dann ist die Figur der Margaret, trotz ihrer archaisch-

44) Tillyard, Shakespeare's history plays (zit. Anm. 17), S. 199.

${ }^{45}$ ) Vgl. ebenda, S. 212.

46) Ebenda, S. 200f.

$\left.{ }^{47}\right)$ Ebenda, S. 204.

48) Shakespeare-Handbuch (zit. Anm. 7), S. 49.

49) Ebenda, S. 346.

50) Vgl. Georg Wilhelm Hegel, Vorlesungen über die Ästhetik, erster und zweiter Teil, mit einer Einführung hrsg. von RüDiger BubNER, Stuttgart 1971, S. $86 f$.

${ }^{51)}$ Ästhetische Grundbegriffe. Historisches Wörterbuch in sieben Bänden, hrsg. von KarLheinz Barck u. a., Stuttgart und Weimar 2003, Bd. 5, S. 394. 
düsteren Dimension, eine ,schöne' Kunstfigur. Wobei das Schöne dem „Anfang“ des „Schrecklichen“" bekanntlich nahe verwandt ist. ${ }^{52}$ )

Im Folgenden soll das Drama auf die hinsichtlich der tektonisch-rhetorischen Funktion des Symmetrie-Prinzips und ihrer normativen Dimension zentralen Stellen durchgesehen werden.

\section{Störung des ordo durch Selbst-Aussonderung: der Rampenmonolog (I/1)}

In 'King Richard III figuriert das zentrale „subject of tragedy ${ }^{\left({ }^{3} 3\right.}$ ) als Iniquity: als ein Subjekt, das die soziale Ordnung und das soziale aequitas-Prinzip durch das Übergewicht eines von sich selbst besessenen Ichs zu sprengen beginnt. ${ }^{54}$ Im Rampenmonolog wird die Selbstausgrenzung Richards mit rhetorischer Eindringlichkeit auf die Bühne gebracht. Anaphorische Wiederholungen des „I“ und des adversativ-oppositionellen „but I“ deuten voraus auf die zumindest ansatzweise tragische Hamartia-Erkenntnis vor dem Untergang des Tyrannen: „Richard loves Richard; that is, I am I" (V/3, V. 184, S. 238). Diese dem Machiavellismus der Renaissance gemäße Identitätsformel${ }^{55}$ ) wird in Shakespeares Königs-Tragödien ausnahmslos mit dem - als notwendig vermittelten - Tode des solitär agierenden ,Spielers' oder Spielleiters bestraft.

Im explizit figuralen Eigenkommentar ${ }^{56}$ ) proklamiert Richard seine Entscheidung zum Bösen: „I am determined to prove a villain“ (I/1, V. 30, S. 8). Trotz der schicksalhaften Determinante körperlicher Verkrüppelung bezeichnet das Wort „determined“ im Kern den frei verantworteten Entschluss zur Absonderung (Sünde) vom sozialen Ganzen: ${ }^{57}$ ) zum Austritt aus dem aequitas-Prinzip des ordo, das jedem seinen für das Ganze zukommenden Platz zuweist, um zu vermeiden, dass die

52) Rainer Maria Rilke, Sämtliche Werke in sechs Bänden, hrsg. vom Rilke-Archiv, in Verbindung mit Ruth Sieber-Rilke besorgt durch Ernst Zinn, Frankfurt/M. 1955ff., Bd. 1, S. 685.

53) Catherine Belsey, The Subject of Tragedy. Identity and Difference in Renaissance Drama, London und New York 1985.

54) Vgl. Elfi Bettinger, King Richard III, in: Interpretationen. Shakespeares Dramen, Stuttgart 2000, S. 38-69, hier: S. 55: „Richards aggressives Insistieren auf seiner Einzigartigkeit, seiner Individualität, verdeutlicht die Sprengkraft dieses ,Subject of Tragedy “.

$\left.{ }^{55}\right)$ Ebenda, S. 65: „Die neue Bedeutung in der Renaissance faßt das Rollenspiel als self-fashioning [vgl. Stephen Greenblatt, Renaissance Self-Fashioning from More to Shakespeare, Chicago 1980], das nun anstatt auf einem festen Platz in der Hierarchie auf der persönlichen Freiheit des autonomen Subjekts zur Selbstverwirklichung insistiert."

56) Vgl. Manfred Pfister, Das Drama. Theorie und Analyse, 11. Aufl., München 2001, S. $251 \mathrm{ff}$.

$\left.{ }^{57}\right)$ Auch nach Ansicht von Clemen „kann hier die äußere Situation nicht verantwortlich gemacht werden für das Verhalten Richards. Die Motivierung für sein Handeln ergibt sich vielmehr allein aus seinem Charakter, seinem Willen" (Clemen, Kommentar, zit. Anm. 5, S. 17). Der Willensentschluss Richards ist demnach „nicht als ,Ich bin bestimmt' im Sinne eines unverschuldet Determiniertseins zu verstehen“ (ebenda, S. 21). 
Zeit ,aus den Fugen' gerät, das soziale Haus seinen Zusammenhalt verliert, weil das Fundament nicht mehr trägt, das Dach ohne Stütze ist, die Fenster und Türen aus ihren Angeln gefallen sind. ${ }^{58}$ ) Wenn der Usurpator Richard den Regenten und den nächsten Thronanwärter ermorden lässt, um als ,Keller' an die Stelle des Giebels zu treten, bewirkt er, dass die Zeit „out of joint ${ }^{\text {“59) }}$ gerät und der soziale Gliederbau an verschiedenen Gelenken ausgerenkt wird. Weniger bildlich gesagt: Gegenüber der durch den Ritus repräsentierten Normativität sozialer ordo tritt Richard als gesetzloser Usurpator und Zer/Störer, als Verkörperung einer verkehrten Welt auf.

Für das Gleichgewicht des Dramas als Gesamtkonzept ist entscheidend, dass der einleitende Planungsmonolog vom Publikum als Verführung zur Komplizenschaft mit Richards exzentrischer Position, also als ein Identifikationsangebot des vice, erkannt wird. ${ }^{60}$ ) Die „frontale Eröffnungsweise ${ }^{\text {“61 }}$ ) hat etwas Überrumpelndes, geradezu Autoritäres an sich und lässt das Publikum in den Sog der Selbstzentrierung Richards geraten.

Etwas anders verhält es sich mit dem beiseite gesprochenen Eigenkommentar „[Aside.] Thus, like the formal Vice, Iniquity, | I moralize two meanings in one word“ (III/1, V. 83, S. 112). Hier entsteht Distanz zur Selbstzentrierung Richards im Rampenmonolog, nicht nur innerhalb der Figur selbst, die sich über den Typus des vice definiert und damit auf die eigene Rollenhaftigkeit, ja Puppenhaftigkeit hinweist, ${ }^{62}$ ) sondern auch innerhalb des Publikums, das, nach Art eines Brechtschen Verfremdungseffekts, aus der fiktionalen Illusion gerissen wird. Dennoch ist die „mit Ironie und Humor gepaarte[n]“ „Kommentatorenrolle“63) Richards auch an dieser Stelle nicht ganz ohne verführerisches Potential. Da Richard seine Mitwisser im Publikum potentiell in die Haltung des „wissenden“, aber auch affektiven „Mitvollzugs“"versetzt, ${ }^{64}$ ) bewirkt er die Möglichkeit der Partizipation „an den Omnipotenzgefühlen des Manipulators" ${ }^{\text {“65 }}$ ) und an der Schadenfreude seiner Überlegenheitskomik. ${ }^{66}$ )

${ }^{58}$ ) Vgl. Tillyard, Shakespeare's history plays (zit. Anm. 17), S. 11: „The Elizabethan conception of world-order was in its outlines medieval although it had discarded much medieval detail. The universe was a unity, in which everything had its place, and it was the perfect work of God. Any imperfection was the work not of God but of man."

59) Shakespeare, Hamlet (I/5) (zit. Anm. 3), S. 108. Vgl. zur Metapher aus den Fugen` und ihrer Funktion innerhalb des frame-of-order-Denkens Suerbaum, Shakespeares Dramen (zit. Anm. 23), S. 99 f.

${ }^{60)}$ Vgl. Shakespeare-Handbuch (zit. Anm. 7), S. 251.

61) Clemen, Kommentar (zit. Anm. 5), S. 24.

62) Vgl. Besnault/Bitot, Historical legacy (zit. Anm. 5), S. 114: „Throughout the first half of the play, he [Richard] indulges in asides that secure our connivance and create the impression that he is both in the world of the play and outside it as a dispassionate Vice-like observer."

$\left.{ }^{63}\right)$ Shakespeare-Handbuch (zit. Anm. 7), S. 345.

64) Ebenda, S. 261.

${ }^{65)}$ Bettinger, King Richard III (zit. Anm. 54), S. 45 f.

$\left.{ }^{66}\right)$ Shakespeare-Handbuch (zit. Anm. 7), S. 261. 
Der Autor als Meta-Spielleiter muss den vice, der im ersten Teil des Dramas die ihm unterlegenen Mitspieler ,vorführt', an späterer Stelle selbst vorführen und als betrogenen Betrüger entwerten sowie töten lassen, um den kunstvoll gestörten ordo wieder zu stabilisieren. Kurz: Er muss Richard, der als „dramaturgischer Motor der Handlung " fungiert, ${ }^{67}$ ) mit einer Antagonistin zusammenprallen lassen, die genügend Anziehungskraft hat, um sowohl das Publikum als auch die anderen Bühnenfiguren in ihren Bann zu ziehen.

\section{Margarets rituelle Verfluchungen (I/3, V. 45-55): \\ Gegengewicht und Gegenmotor}

Mit Margaret wird Richard eine „echte Gegenkraft“",gegenübergestellt.“" ${ }^{\text {“8 }) ~ I h r ~}$ erster Auftritt (in I/3) „beherrscht die Szene noch mehr als Richards Gegenwart und läßt zum ersten Mal eine Richard ebenbürtige Gestalt in Erscheinung treten ${ }^{(69}$ ) Das Kontinuum des Dramas wird durch den Antagonismus von Richard und Margaret geprägt und strukturiert, als wäre sie die heimliche Königin die Königin eines Schattenkabinetts des Schicksals. Die Intention des Autors beschränkt sich demgemäß keineswegs darauf, Richard im Einklang mit der Tudor-Geschichtsschreibung „als Erzbösewicht zu zeichnen “; ${ }^{\text {(70) }}$ vielmehr kommt es Shakespeare darauf an, diesen „Erzbösewicht", den vice mit all seiner verfänglichen Attraktionskraft, ${ }^{71}$ ) darstellerisch dadurch auszubalancieren, dass er ihm ein starkes Gegengewicht in der Figur Margarets beigegeben hat, deren sakrale Aufwertung schwerlich als bloßes Votum für das Haus Lancaster und damit als indirektes Fürstenlob des Hauses Tudor zu deuten ist.

Stellt man die Frage nach dem ,Rituop', dem sozialen Ort, der sozialen Situation des Klage- und Verfluchungs-Rituals, das Margaret in I/3 ausführt, so ist das Rituop durch Isolation und Devianz der fluchenden Person bei gleichwohl beträchtlicher Wirkkraft geprägt: In einem „Raum im Palast“ (S. 35) befinden sich Königin Elizabeth, die späteren Richard-Opfer Rivers und Grey, der OpferKomplize Buckingham, das Opfer Hastings, Dorset sowie Stanley, einer der wenigen, der Richard rechtzeitig genug durchschaut, um sich retten und den Gegnern Richards Hilfe leisten zu können. Margaret ist unter diesen Adligen isoliert, doch gerade durch die initiale Isolation ergibt sich ein gigantisches szenisches Ent-

${ }^{67}$ ) Ebenda, S. 345.

${ }^{68}$ ) Clemen, Kommentar (zit. Anm. 5), S. 71.

${ }^{69)}$ Ebenda.

${ }^{70}$ Shakespeare-Handbuch (zit. Anm. 7), S. 344

$\left.{ }^{71}\right)$ Vgl. ebenda, S. 278: „das ,Vice', das in den Moralitäten das menschliche Laster verkörpert. Mit seiner unmotivierten Freude an der Bosheit, seiner grotesken Komik, seinem Hang zu histrionischen Gesten und zu publikumsbezogenem Reden und Agieren eignen ihm besonders theaterwirksame Attribute." 
wicklungspotential: ${ }^{72}$ ) Die abgesetzte und verbannte Königin, die zuletzt, kraft der Erfüllung ihrer Flüche, einen für ihre Kontrahenten unerwarteten Einfluss üben wird, gelangt schon innerhalb von I/3 aus einer marginalisierten in eine gewichtige Position, aus der heraus sie eine geradezu magische Gewalt über die Seelen der ihr scheinbar Überlegenen ausübt. ${ }^{73}$ ) „My hair doth stand on end to hear her curses“ (I/3, V. 304, S. 55): So bringt, unter Verwendung des emphatic do, das spätere Terror-Opfer Hastings die starke Wirkung des Fluchs zum Ausdruck. Damit antizipiert er seine Metanoia im Angesicht des Todes, während er Margaret kurz zuvor noch als „falsch weissagende Frau“ beschimpft und aufgefordert hatte, ihren „rasenden Fluch“ zu beenden (V. 247, S. 51). Die Wirkungsmacht Margarets, der Frau im Off, ihre ritual efficacy, ${ }^{74}$ ) die „Handlungsmacht (agency) ${ }^{“ 75}$ ) ihrer rituellen Verfluchungen, ist stärker als die Abwehr der anwesenden Lords zusammen. Margarets Verfluchungen stellen, trotz aller Abwehr, einen Akt dar, dessen „Bedeutsamkeit“ sich sowohl die Akteurin als auch die „Zuschauer“ „bewußt sind“"76) mithin ein Ritual mit Angst erzeugender Wirkungsmacht. Solche Wirkungsmacht erweist Margaret als heimliche Königin, die hinter die Kulissen zu blicken vermag und, trotz aller Widerstände, die ihr das Haus York und dessen Höflinge entgegenbringen, ihren Macht-An-Spruch inmitten scheinbarer Ohnmacht behauptet. Diese Kraft der Selbstbehauptung aktiviert Margaret durch den wirkmächtigen speech act des Fluchens, der sich, sofern man an die Wirkmächtigkeit glaubt, als deklarativer Sprechakt (,hiermit verfluche ich Hastings'), ansonsten als expressiver, partiell auch als direktiver Sprechakt klassifizieren lässt. In jedem Fall does she do "something“ (III/1, V. 193, S. 120) with her words: Sie tötet diejenigen, deren unnatürlichen Tod sie prophezeit, verbal. Ist ein Fluch doch nichts anderes als ein

72) Clemen, Kommentar (zit. Anm. 5), S. 72: Ein vorbereitender „Polylog“ macht ihren [Margarets] Auftritt desto wirkungsvoller. Dieser verdeutlicht zudem: „Richard Gloucester steht keine geschlossene Front gegenüber, sondern eine durch gegenseitige Missgunst, durch Haß und Neid gespaltene Hofgesellschaft; und diese Schwäche wird er ausnützen.“

73) Vgl. Phyllis Racin, Women's Roles in the Elizabethan History Plays, in: The Cambridge companion to Shakespeare's history plays, hrsg. von Michael Hattaway, Cambridge 2004, S. 5-85, hier: S. 77: „Both Joan and Margaret, for instance, have a powerful theatrical presence that challenges the dominance of the heroic English men who are featured in the represented history. In Richard III, although it is Richmond, rather than any of the women, who finally puts an end to Richard's bloody career, the women have far more time on stage, challenging Richard's theatrical dominance in a way that Richmond never manages to do. Moreover, it is Margaret and Joan, rather than any of the male characters in the earlier plays, who anticipate Richard's demonic energy, his transgressive, irreverent wit, and his vivid theatrical presence. The fact that all three play villainous roles in the represented action in no way diminishes the powerful appeal they possess in performance".

74) Dücker, Rituale (zit. Anm. 8), S. 61.

${ }^{75}$ ) Ebenda.

76) Vgl. die oben bereits zitierte Definition von Wolfgang Braungart, Ritual und Literatur (zit. Anm. 19), S. 4 und S. 6. 
im Affekt des Zornes ausgesprochener Unheilswunsch, der einem Menschen oder einer Sache Vernichtung oder Schaden bringen soll; ursp. ein Zauberwort, das aus eigener Kraft wirkt, später Herabrufung göttlicher Strafe oder Weihung von Feinden an unterirdische Mächte. Zu feierl. Ritual erhoben, wächst aus dem Fluch die öffentl. Verdammung, etwa im Anathema des kirchlichen Bannes. ${ }^{77}$ )

Die ursprüngliche Wirkkraft des Zauberworts vermag die als „f[F]oul wrinkled witch " (I/3, V. 164, S. 46) beschimpfte, an die Tradition mächtiger Totenbeschwörerinnen wie der alttestamentlichen ,Hexe von En-Dor erinnernde (1 Sam 28, 3-24) Margaret zu revozieren:

Though not by war, by surfeit die your king,

As ours by murder, to make him a king!

Edward, thy son, that now is Prince of Wales,

For Edward, my son, which was Prince of Wales,

Die in his youth by untimely violence!

Thyself a queen, for me that was a queen,

Outlive thy glory, like my wretched self! [...]

Rivers, and Dorset, you were standers by, -

And so wast thou, Lord Hastings, - when my son

Was stabb'd with bloody daggers: God, I pray him,

That none of you may live your natural age,

But by some unlook'd accident cut off. (V. 197-214, S. 48)

Der durch starke Rhetorizität und magische $\mathrm{Wucht}^{78}$ ) beherrschte, daher als ritushaft anzusehende Fluch-Akt Margarets wiederholt stilistisch, worum es inhaltlich geht: „repetition of what thou hast marr'd“ (I/3, V. 165, S. 46). Figuren der Wiederholung wie Epipher („die your king | [...] make him a king“",that now is Prince of Wales, | [...] which was Prince of Wales“), Epanalepse („Thyself a queen, for me that was a queen“) variieren das alttestamentliche Talionsgesetz „Auge um Auge, Zahn um Zahn“ (Mt 5, 38). König um König, Prinz um Prinz (als soziale Figuren mit höchstem Wert), ein Edward (als Individuum mit bestimmtem persönlichen Wert) für einen anderen Edward (mit einem anderen persönlichen Wert).

Unerbittlichkeit der Sühne, die Gleiches für Gleiches einfordert, kommt sprachlich darin zum Ausdruck, daß die Verwünschungen mehrfach als Gleichungen formuliert sind, bei denen (unter Verwendung der Epipher) Gleiches sich in strenger Entsprechung und in einprägsamer Echowirkung in gegensätzlichem Sinne gegenübersteht. ${ }^{79}$ )

Jeder Vers ist nach dem Prinzip der Symmetrie gebaut; die Verse Margarets in ihrer Aufeinanderfolge (auch in IV/4) sind ein extensiver parallelismus membrorum,

$\left.{ }^{77}\right)$ Dtv-Lexikon in 20 Bänden (zit. Anm. 37), Bd. 6, S. 16.

$\left.{ }^{78}\right)$ Magische Wörter und Fluch-Wörter gehören zur Gruppe der words of power, also der Lexeme mit starkem „Emotionspotenzial“" (Monika Schwarz-Friesel, Sprache und Emotion, Tübingen und Basel 2007, S. 3).

${ }^{79}$ ) Clemen, Kommentar (zit. Anm. 5), S. 87. 
der das Symmetrieprinzip auf einer höheren strukturellen Ebene wiederholt und gleichzeitig den alttestamentlichen Charakter der Figur profiliert. Johann Gottfried Herder stellt den Parallelismus in seiner Schrift ,Vom Geist der ebräischen Poesier (1781/82) als alttestamentliche Urform der Dichtung dar, die der Rede des Affekts besonders gemäß sei:

Sobald sich das Herz ergießt, strömt Welle auf Welle, das ist Parallelismus. Es hat nie ausgeredet, hat immer etwas neues zu sagen. Sobald die erste Welle sanft verfließt, oder sich prächtig bricht am Felsen, kommt die zweite Welle wieder. Der Pulsschlag der Natur, dies Othemholen der Empfindung ist in allen Reden des Affekts, und Sie wolltens in der Poesie nicht, die doch eigentlich Rede des Affekts sein soll? ${ }^{80}$ )

Das Talionsprinzip der Verse Margarets erzeugt aber nicht allein einen alttestamentlichen, sondern zudem einen griechisch-antiken, chorischen Effekt. Clemen zufolge ist Margaret „diejenige Figur, die am stärksten die Funktionen des Chorus der klassischen Tragödie weiterführt“" ${ }^{\circ 1}{ }^{1}$ „,Doch ist sie gleichzeitig eine von $\mathrm{Haß}$ und Schmerz erfüllte Greisin, die noch genug Menschliches an sich hat, um nicht etwa als bloß betrachtende und unbeteiligte neutrale Chorusfigur zu wirken. ${ }^{\text {"82 } 2)}$

Chorisch wirkt die Figur der Margaret insofern, als ihr in den asides eine auf das Publikum bezogene Kommentarfunktion zukommt. Darüber hinaus hat sie eine mahnend-warnende Funktion in Bezug auf die mit ihr anwesenden Bühnenfiguren. Auch ist die Musikalität ihrer Sprache unverkennbar. Hinzu kommt: „Margaret greift nicht in die Handlung ein und steht selber außerhalb der Handlung. ${ }^{(83)}$ )

$\mathrm{Zu}$ dieser Position außerhalb der Handlung gehört, dass das Talionsprinzip der Verse Margarets eine Art Fermate inmitten der auf die Katastrophe zurasenden dramatischen Zeit bildet. Diese rasende Zeit wird, so scheint es, durch Gottes heilige Zeit angehalten - in der Naxos-Einspielung des Dramas mit Kenneth Branagh und Geraldine McEwan wird dieser Effekt dadurch zum Ausdruck gebracht, dass Margarets Flüche und Klagen in I/3 und IV/4 mit dem Geläut einer Kirchenglocke untermalt werden. Aus dem Zeit-Stillstand reißt der Crash Margarets und Richards punktuell heraus: „Glou. Margaret! | Queen Marg. Richard!“ (I/3, V. 234f., S. 50).

${ }^{80}$ Johann Gottraied Herder, Vom Geist der Ebräischen Poesie I, zit. nach Ders., Schriften zum Alten Testament, hrsg. von Rudolf SMend (= Werke in zehn Bänden, hrsg. von Martin Bollacher u. a., Bd. 5), Frankfurt/M. 1993, S. 686.

${ }^{81}$ ) Clemen, Kommentar (zit. Anm. 5), S. 78.

82) Ebenda, S. 79.

${ }^{83}$ ) Ebenda. Vgl. Besnault/Bitot, Historical legacy (zit. Anm. 5), S. 117: „Whereas Richard initiates and propels the action forward, Margaret structures it and she orchestrates it. When she appears on stage, in 1.3 and 4.4, she introduces suspensions of activity, changes of focus and of tone. When offstage, she brings remembrance of her prophetic curses as their victims succumb to their doom." 
Dieser der Stichomythie ähnliche Crash ist keine Dissonanz, die aus dem Drama der Symmetrie herausfiele, sondern ist das Drama in nuce: ein kunstvoller Knall-Effekt, getragen, im Gesamtdrama ausbuchstabiert und ausbalanciert von der Spannkraft des Autors.

\section{Rituelle Totenklage (II/2)}

Im zweiten und dritten Akt ist die Figur der Margaret abwesend. Dafür erhalten andere Vertreterinnen und Vertreter der Opfergruppe eine Stimme: Richards Mutter, die Herzogin von York, ihre Enkel, der Sohn und die Tochter von Clarence, sowie die verwitwete Königin Elizabeth treten in der zweiten Szene des zweiten Akts gemeinsam auf, um das in I/3 begonnene Klageritual fortzuführen:

Queen Eliz.

Give me no help in lamentation;

I am not barren to bring forth complaints:

All springs reduce their currents to mine eyes,

That I, being govern'd by the watery moon,

May send forth plenteous tears to drown the world!

Ah! for my husband, for my dear Lord Edward!

Children.

Ah! for our father, for our dear Lord Clarence!

Duch.

Alas! for both, both mine, Edward and Clarence!

Queen Eliz.

What stay had I but Edward? and he's gone.

Children.

What stay had we but Clarence? and he's gone.

Duch.

What stays had I but they? and they are gone.

Queen Eliz.

Was never widow had so dear a loss.

Children.

Were never orphans had so dear a loss. Duch.

Was never mother had so dear a loss.

Alas! I am the mother of these griefs:

Their woes are parcell'd, mine are general.

She for an Edward weeps, and so do I;

I for a Clarence weep, so doth not she:

These babes for Clarence weep, and so do I;

I for an Edward weep, so do not they:

Alas! you three, on me, threefold distress'd,

Pour all your tears; I am your sorrow's nurse,

And I will pamper it with lamentation. (II/2, V. 66-88, S. 92) 
Auch in dieser Szene dienen Figuren der repetitio der eindringlichen Beklagung des durch Richard bewirkten Leids, darunter die Epanalepse („for my husband, for my dear Lord Edward!“, „for our father, for our dear Lord Clarence!“), die Geminatio („for both, both mine, Edward and Clarence!“) und die Anapher bzw. Epipher („What stay had I but Edward? and he's gone. | What stay had we but Clarence? and he's gone. | What stays had I but they? and they are gone." etc.).

In der starken Stilisierung und Formelhaftigkeit dieser Klagestellen bloß überspitzte akademisch-rhetorische Kunstübung zu sehen, reicht als Erklärung nicht aus. Unverkennbar ist, daß dieser Sprachstil mit seiner Neigung zu Symmetrie und Gleichklang, zu respondierender Echowirkung und variierender Wiederholung, dem Eindruck eines gemeinsamen feierlichen Rituals dienen soll. ${ }^{84}$ )

Die Wiederholungen und Parallelismen sind ja nicht nur kunstvolle sprachliche Figur, sondern hämmern uns die Gleichartigkeit, Wiederkehr und Häufung des Leids durch Klang und Rhythmus ein, die ständige Wiederholung der Namen von Edward und Clarence [...] hat eine magisch-beschwörende Wirkung. ${ }^{85}$ )

Formelhaftigkeit und Ritualhaftigkeit müssen als Korrelation gesehen werden: Rituelle Texte sind per se "formelhaft“. „Als ein gemeinsames Merkmal gilt das Maß ihrer Fixiertheit, anders gesagt: das Maß ihrer Formfestigkeit, also dessen, was der Begriff des Formelhaften andeutet." ${ }^{86)}$ Im eminenten Maße formfest sind Zauberformeln, deren Wirkungsabsicht u.a. darin besteht, Lebewesen kraft der Fixiertheit der Sprache fest zu bannen und ggf. in unbewegliche Objekte zu verwandeln. Freilich wirkt ein solcher Zauber nur, wenn sich das affect attunement der zu verzaubernden Personen davon tangieren lässt. So ist auch die Wirkung des Klagerituals der Szene II/2 und der rituellen Verfluchung von I/3 davon abhängig, dass affektive Abwehrschranken (so in der Szene I/3) durchbrochen werden und das Mitleid (eleos) des Publikums geweckt wird (so in der Szene II/2). Nicht selten ist das Rituelle

aufs engste mit einer den schriftlichen Texten und mündlich tradierten Sprüchen unterstellten kausalen Kraft, einem magischen oder energetischen Wirkpotential verbunden, dem die Mitspieler unter bestimmten gesellschaftlichen Bedingungen einen Anspruch auf Wahrheit - eine, formelhafte Wahrheit - zugestehen. ${ }^{87}$ )

Ein ritueller Sprachstil, dessen Funktion darin besteht, Bühnenfiguren und Publikum für das ,energetische Wirkungspotential' geformter Verse zu öffnen, tendiert in seiner starken Abweichung von der Alltagssprache zum Funktionalstil (Funktiolekt) und erinnert an jene Call-and-Response- oder Responsorien-

84) Clemen, Kommentar (zit. Anm. 5), S. 148.

$\left.{ }^{85}\right)$ Ebenda, S. 149

${ }^{86)}$ Hardt, Rituale (zit. Anm. 23), S. 30.

87) Ebenda, S. 32. 
Gesänge der Liturgiefeier, deren Aufgabe darin besteht, die Affektlage von Priester/in oder Vorbeter/in einerseits und der Gemeinde andererseits in Übereinstimmung zu bringen.

In IV/4 erreicht das von Tillyard, Clemen und Anderen beschriebene, religiös „stilisierte Pathos der Sprache“ seine höchste Stufe: ${ }^{88}$ )

the full religious temper of the play only comes out in the two great scenes in the last third of the play: the lamentations of the three queens after Richard has murdered the princes in the Tower, and the ghosts appearing to Richard and Richmond before Bosworth. [...] Both scenes are ritual and incantatory to a high degree, suggesting an ecclesiastical context; both are implicitly and explicitly pious; and both are archaic, suggesting the prevalent piety of the Middle Ages. The incantation takes the form not only of an obvious antiphony like Queen Margaret's balancing of her own woes with Queen Elizabeth's - [...] - but of a more complicated balance of rhythmic phrases and of varied repetitions, as in the Duchess of York's self-address. ${ }^{89}$ )

In kanonartigem Wechsel stimmt jede der Beteiligten ihre Klage an; das gemeinsame Thema ist gleichsam auf mehrere Stimmen verteilt, die teils sich kontrapunktisch gegenüberstehen, teils vereinigt werden..$^{90}$ )

\section{Margaret, Königin Elizabeth, Herzogin von York: finale Totenklage und gebrochene Solidarität weiblicher Opfer (IVI4)}

Einem cantus firmus oder Ostinato gleich, wiederholt das Drama Margarets Flüche und das Talionsprinzip, nach dem in der Logik der Flüche Richards Mordopfer verrechnet werden. Unter einem Ostinato versteht die Musikwissenschaft ein beständig wiederholtes Strukturelement „- ob harmonische Folge, Melodie- oder Baßformel -“, das als „unveränderlicher Kernpunkt wahrgenommen“ wird. $\left.{ }^{11}\right)$ Die etymologische Verwandtschaft von ,Ostinato und ,Obsession' gibt bereits zu verstehen, dass das Ostinato, das „ursprünglich auf rituelle Bereiche wie ,Tanz' oder ,religiöse Ekstase ' bezogen war", ${ }^{\text {(92) }}$ eine „,eigenartige Suggestivkraft" zu entfalten pflegt: ${ }^{93}$ ) etwas „Magisch-Bezwingendes“ ${ }^{(94}$ ) dessen Wirkung sich nicht voll erschließen lässt. Gleichzeitig wird das Ostinato, ähnlich wie das an die Figur

88) Clemen, Kommentar (zit. Anm. 5), S. 251.

${ }^{89}$ ) Tillyard, Shakespeare's history plays (zit. Anm. 17), S. $204 \mathrm{ff}$.

${ }^{90}$ ) Clemen, Kommentar (zit. Anm. 5), S. 251.

${ }^{91}$ ) Metzler Sachlexikon Musik. Auf der Grundlage des von Günther Massenkeil hrsg. Großen Lexikons der Musik (1978-1982/1987), red. Bearbeitung der Neuausgabe: RaLF Noltensmeier, Stuttgart und Weimar 1998, S. 783.

92) Christine Lubkoll, Mythos Musik. Poetische Entwürfe des Musikalischen in der Literatur um 1800, Freiburg 1995, S. 227.

${ }^{93}$ ) Metzler Sachlexikon Musik (zit. Anm. 91), S. 783.

94) Ebenda. 
der Margaret gekoppelte Strukturprinzip von ,King Richard III, als „festgefügtes Bauelement, d. h. als rationale Struktur, wahrgenommen. ${ }^{(95)}$ ) Die spezifische Wirkung des Ostinatos gründet in dem ,schwebenden Gleichgewicht zwischen diesen

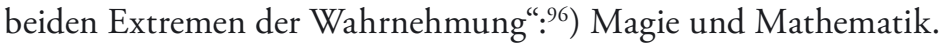

Zum kunstvoll berechneten Arrangement der symmetrischen Tektonik des Dramas gehört, dass Margaret exakt zweimal auftritt: in den Szenen I/3 und IV/4. Dabei kommt der Szene des ersten Akts typologisch die Funktion der figura, der Szene des vierten Akts die Funktion der adimpletio $\mathrm{zu}^{97}$ ) - um „dem Funktionsschema von Urbild und Abbild zu genügen, muss die entsprechende Handlung mindestens zweimal aufgeführt worden sein.“" ${ }^{(98}$ ) Von „Mustern der Präfigurierung und Wiederholung" ist ,King Richard III insgesamt stark geprägt. ${ }^{99}$ )

Margaret beginnt ihren Auftritt der Szene IV/4, ,in dem sich die Essenz des ganzen Dramas verdichtet ${ }^{1100}$ ), mit einem Kommentar zum Leid der Yorks, der (analog zur Szene I/3) zunächst allein vom Publikum gehört wird: „right for right“ (IV/4, V. 15, S. 184), „Plantagenet doth quit Plantagenet; | Edward for Edward pays a dying debt" (IV/4, V. 20, S. 184).

Auf die anklagende Frage Elizabeths (V. 21-24), warum Gott die Ermordung ihrer Prinzen zugelassen habe, wann er bei einem solch grausamen Verbrechen jemals geschlafen habe, antwortet Margaret, ebenfalls klagend: „When holy Harry died, and my sweet son" (V. 25, S. 184). Nach Margarets Verständnis von göttlicher Gerechtigkeit muss Gott, wenn er die Ermordung eines das Gottesgnadentum verkörpernden, mithin , heiligen' Königs zulässt, zum Ausgleich ein Opfer auf der Seite der Feinde fordern, die den Mord verschuldeten. Ist dieser unversöhnliche Geist des Talionsgesetzes, schön' im Sinne von ahd. fagari, schön im Sinne eines Vorscheins göttlicher Gerechtigkeit? Aus alttestamentlicher Sicht oder aus der Sicht einer aristokratischen Ethik der Blutrache sehr wohl, nicht jedoch aus neutestamentlicher Sicht.

Vielleicht ist Shakespeares „hybrid play“,King Richard III ', das Elemente der Historie, der Moralität und der Rachetragödie (nach Seneca bzw. Kyd) ${ }^{101}$ ) verbindet, nicht zuletzt darin history play, dass es die im englischen Adel des 16. und 15. Jahrhunderts realgeschichtlich vorherrschende Ideologie der Blutrache stärker gewichtet als die überzeitliche Maxime christlicher Feindesliebe. Mit anderen

95) Ebenda.

96) Ebenda.

${ }^{97}$ Clemen, Kommentar (zit. Anm. 5), S. 248: „Margaret war seit I,3 im Drama nicht mehr aufgetreten. Ihr Wiederauftauchen nach einer so langen Pause und ihr unmittelbar folgendes endgültiges Abtreten aus dem Stück“ verleihen ihrem Auftritt eine besondere Wirkungsmacht.

${ }^{98)}$ DüCKer, Rituale (zit. Anm. 8), S. 42.

$\left.{ }^{99}\right)$ Shakespeare-Handbuch (zit. Anm. 7), S. 346.

${ }^{100}$ ) Clemen, Kommentar (zit. Anm. 5), S. 250.

101) Besnault/Bitot, Historical legacy (zit. Anm. 5), S. 120. 
Worten: Innerhalb des Gottesbilds einer realgeschichtlichen Hocharistokratin wie Queen Margaret von Anjou (I429-I482) bilden Blutrache und dynastische Feindschaft keine Fremdkörper, sondern sind theologisch legitimierbar über die vorgebliche Legitimität bzw. Illegitimität des jeweiligen Herrscherhauses. „Holy Harry“ war ,God's own king., God's own curse“ trifft, allen voran, den Usurpator Richard III, aber auch jene teils schuldigen, teils unschuldigen Adligen des Hauses York, die von der Ermordung , holy Harrys' teils absichtlich, teils unabsichtlich profitierten bzw. profitiert hätten - wie die Söhne Elizabeths, sofern sie an die Regentschaft gelangt wären. Indem Shakespeare gerade diese Söhne als geborene Könige ikonisiert, würdig und liebenswert, geistreich und dezidiert genug, um sich als „most royal“ zu erweisen (to prove most royal vs. to prove a villain), ${ }^{102}$ ) zeigt er die einseitige Verbohrtheit der Perspektive Margarets auf. Nichtsdestotrotz wählt er gerade diese starke Figur und nicht die zarten Prinzen, um ein Gegengewicht zu der Figur Richards aufzubauen, welches das gesamte Drama, ja die gesamte Rosenkrieg-Tetralogie durchzieht.

Ist diese tektonische Entscheidung ästhetizistisch, insofern sie sich über das Unmoralische in der Margaret-Figur hinwegsetzt, um sie ästhetisch zu rechtfertigen (über repetitio, Strukturprinzipien wie Symmetrie und Parallelismus sowie über den großen rhetorischen Aufwand, mit dem das Drama ihre Auftritte in Szene setzt)? Ästhetizistisch, insofern die Namen der Ermordeten, der These Warren Chernaiks zufolge, ${ }^{103}$ ) gleich Perlen einer kostbaren Kette entindividualisiert und somit dehumanisiert werden? ${ }^{\text {104 }}$ ) Wenn ja, müsste man wohl die gesamte AdelsIdeologie der Entindividualisierung zeihen, denn im Kern geht es dem Adel um gens, also soziale Identität, nicht um das Prinzip unverwechselbarer Individualität. Gloucester ist in erster Linie Vertreter seiner Grafschaft, erst in zweiter Linie das jeweilige historische Individuum, desgleichen Buckingham, Leicester und die anderen Adelsvertreter.

Vielleicht aber ergibt sich der nivellierende Effekt eher daraus, dass Margaret von einem übergeordneten Standpunkt aus spricht, beinahe einem göttlichen, der zu ihrer Allwissenheit in I/3 passt. Demnach wäre der entindividualisierende Effekt ihrer Klagen und Flüche der Perspektive des obersten Weltenrichters geschuldet, der jeden „cause to mourne“ (IV/4, V. 33, S. 186) als gleichwertig gewichtet und ein unschuldiges Mordopfer der Yorks nicht anders beurteilen darf als ein unschuldiges Mordopfer der Lancasters. Aus dieser göttlichen Perspektive,

102) Shakespeare, Hamlet (zit. Anm. 3), S. 312.

${ }^{103}$ ) Chernaik, The Cambridge Introduction to Shakespeare's History Plays (zit. Anm. 32), S. $62 \mathrm{f}$.: "there is no sense of divine justice in this endless cycle of revenge, in which even the names of the dead lose their individuality."

104) Hardt, Rituale (zit. Anm. 23), S. 43, verweist auf Forschungsansätze, wonach die Formalisierungstendenz des Rituellen generell „zur Entleerung der propositionalen Gehalte“ und damit zu einer unkritischen Abstraktion von Inhalten führt. 
der sich die drei ,Klageweiber' der Szene, Margaret, Elizabeth und die Herzogin von York, in letzter Instanz unterordnen, wird, trotz unüberwindlicher Rivalität, eine gewisse „society“ (IV/4, V. 38, S. 186) der Leidenden möglich, die sich auch körperlich manifestiert. ${ }^{105}$ ) Gleichwohl entspricht es dem Stil des nun folgenden „Klage-Wechselgesangs, daß die eigentliche Dialogbeziehung fehlt. ${ }^{106}$ ) Stattdessen stellt sich eine indirekte Dialogizität durch die gemeinsame Adressierung Gottes als der letzten Instanz Gerechtigkeit suchender Leidender her.

Sitting down by her [Queen Eliz.]

Queen Marg. If ancient sorrow be most reverent,

Give mine the benefit of seniory,

And let my griefs frown on the upper hand.

If sorrow can admit society.

Sitting down with them.

Tell o'er your woes again by viewing mine:

I had an Edward, till a Richard kill'd him;

I had a Harry, till a Richard kill'd him;

Thou hadst an Edward, till a Richard kill'd him;

Thou hadst a Richard, till a Richard kill'd him.

Duch.

I had a Richard too, and thou didst kill him;

I had a Rutland too, thou holp'st to kill him.

Queen Marg.

Thou hadst a Clarence too, and Richard kill'd him. (V. 34-46, S. 186)

Auf sieben Verse - eine heilige Zahl - dehnt Shakespeare eine rituelle Klage aus, die im jeweils ersten, anaphorischen Teil des Verses Totenklage ist, im jeweils zweiten, mit Epiphern gestalteten Teil des Verses eine gebündelte Anklage des Mörders vorträgt. ${ }^{107}$ ) Der „memoria-Aspekt des Rituals ${ }^{\text {“108) }}$ geht somit einher mit

${ }^{105}$ ) Vgl. Clemen, Kommentar (zit. Anm. 5), S. 261: „die drei Frauen lassen sich in gleichen Abständen auf dem Boden nieder, so daß auch der Bühnenvorgang stilisiert erscheint, was von den Klageszenen der attischen Tragödie nicht gesagt werden kann."

${ }^{106)}$ Ebenda, S. 251

${ }^{107)}$ Ebenda, S. 252: „Die vielfachen Verkettungen und Entsprechungen, in welche die einzelnen Verszeilen und Aussagen durch die ineinandergreifende Verwendung der Stilfiguren gebracht werden, sind Zeichen für die Verkettung der Schuldzusammenhänge. Die zahlreichen Wiederholungsfiguren sind Ausdruck für die Wiederkehr des Gleichen, für den unentrinnbaren Kreislauf der einmal verübten bösen Tat, die fortzeugend Böses gebiert. [...] Die Verbindung von Anapher [,Where/where“; „for/for"] und Epipher z. B. in den Versen 39-46 verdeutlicht den Gleichlauf, die Vergeltung und die unheimliche Wiederkehr der immer wieder auf Richard zurückweisenden Verbrechen. Die siebenfache Wiederholung der Endworte „kill'd him“ bzw. „kill him“ in sieben rhythmisch gleich gebauten Versen, in denen die Namen immer an denselben Stellen stehen und die gleichen Bedeutungen tragen, ist von einhämmernder Wirkung."

$\left.{ }^{108}\right)$ Gerrit Jasper Schenk, Einleitung: Tradition und Wiederkehr des Rituellen, in: Ritualdynamik. Kulturübergreifende Studien zur Theorie und Geschichte rituellen Handelns, hrsg. 
der Forderung nach göttlicher Gerechtigkeit, die den „Höllenhund“ Richard endlich zu Fall bringen soll.

From forth the kennel of thy womb hath crept

A hell-hound that doth hunt us all to death:

[...]

O! upright, just, and true-disposing God,

How do I thank thee that this carnal cur

Preys on the issue of his mother's body,

And makes her pew-fellow with others' moan. Duch.

O! Harry's wife, triumph not in my woes:

God witness with me, I have wept for thine.

Queen Marg.

Bear with me; I am hungry for revenge,

And now I cloy me with beholding it.

Thy Edward he is dead, that kill'd my Edward;

Thy other Edward dead, to quit my Edward;

Young York he is but boot, because both they

Match not the high perfection of my loss:

Thy Clarence he is dead that stabb'd my Edward;

And the beholders of this tragic play,

The adulterate Hastings, Rivers, Vaughan, Grey,

Untimely smother'd in their dusky graves,

Richard yet lives, hell's black intelligencer,

Only reserv'd their factor, to buy souls

And send them thither; but at hand, at hand,

Ensues his piteous and unpitied end:

Earth gapes, hell burns, fiends roar, saints pray,

To have him suddenly convey'd from hence.

Cancel his bond of life, dear God! I pray,

That I may live and say, The dog is dead. (V. 47-78, S. 186-188)

Die Singularität Richards inmitten einer Reihe schuldig-unschuldiger Opfer bzw. Opfer-Täter wird durch die Formel „hell's black intelligencer" scharf herausgemeißelt. Den Machenschaften dieses Geheimdienst-Agenten der Hölle muss schleunigst ein Ende gemacht werden (eine Dringlichkeit, die durch die Geminatio „at hand, at hand" rhetorisch vermittelt wird). Die asyndetische Bündelung von vier wuchtigen Kurzsätzen in einem einzigen Vers („Earth gapes, hell burns, fiends roar, saints pray"), die syntaktisch völlig aus dem Kontext fällt, bringt mit apokalyptischen Bildern die Erwartung zum Ausdruck, dass Himmel und Hölle den schwarzen Richard bald in den Abgrund reißen werden.

von Dietrich Hardt und Gerrit Jasper Schenk, Heidelberg 2004, S. 11-26, hier: S. 13 (unter Verweis auf Jan Assmann, Das kulturelle Gedächtnis. Schrift, Erinnerung und politische Identität in frühen Hochkulturen, München 1999). 
Nach diesem Abgesang folgt ein kunstvolles Echo auf die erste ,Antiphon', das erste von Queen Margaret und der Herzogin von York vorgetragene ,Responsorium': In wiederum sieben rhetorischen Fragen, die das biblische Ubi sunt principes (Bar 3,16-19) variieren, ${ }^{109}$ ) wird Königin Elizabeth nach dem Verbleib ihrer „dignity“ gefragt, wobei die alliterierenden Fragewörter „where“, „wherein“ und „who“ durch Anaphern bzw. durch Epanalepsen scharf herausstechen.

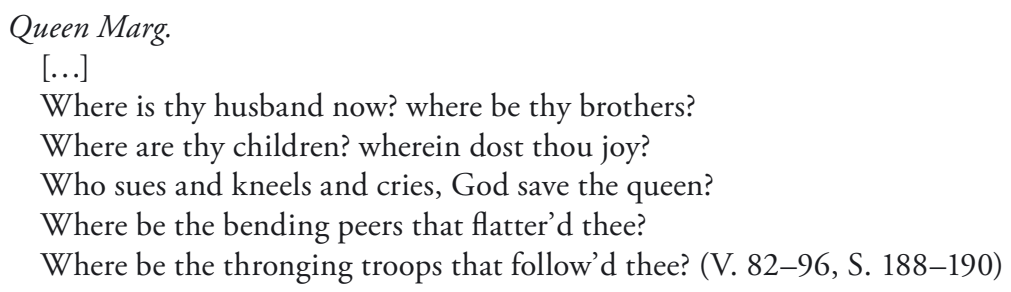

In abermals sieben anaphorisch gesetzten Versen, die eine Kette von Antithesen bilden, zeigt Margaret auf, was aus der tief gefallenen Königin geworden ist: ${ }^{110}$ )

For happy wife, a most distressed widow:

For joyful mother, one that wails the name;

For one being sued to, one that humbly sues;

For queen, a very caitiff crown'd with care;

For she that scorn'd at me, now scorn'd of me;

For she being fear'd of all, now fearing one;

For she commanding all, obey'd of none. (V. 98-104, S. 190)

Aus einer hoch erhobenen Königin wurde eine tief gestürzte: $\left.{ }^{111}\right)$ „Thus hath the course of justice whirl'd about" (IV/4, V. 105, S. 190).

Deutet man die Szene IV/4 als „enactment of a tragic rite“, als Wechselgesang von drei ,Klageweibern', die aufgrund der liturgischen Tonart ihrer Verse auch mit den drei Marien eines geistlichen Spiels des Mittelalters in Verbindung gebracht werden können, ${ }^{112}$ ) so muss gleichwohl der Unterschied zwischen der rituellen

${ }^{109)}$ Clemen, Kommentar (zit. Anm. 5), S. 262.

$\left.{ }^{110}\right)$ Ebenda, S. 252: „Die Antithesen, die in paralleler Anordnung in Margarets Reden (bes. S. 97ff.) häufig erscheinen, sind adäquates Sprachmittel für den von ihr in solcher Form geschauten Gegensatz zwischen Einst und Jetzt, Schein und Sein, Emporgehobensein und Erniedrigung."

111) Shakespeare, Richard III (zit. Anm. 30), S. 750.

${ }^{112}$ ) Vgl. Clemen, Kommentar (zit. Anm. 5), S. 258: Vorstufe der Klageszenen seien u. a. „die liturgischen Szenen des Mittelalters und die Marienklagen [drei Frauen, drei Marien] der Mystery Plays des 14. und 15. Jahrhunderts. Auch hier vereinigen sich (meist drei) klagende Frauen zu einem Wechselgesang, bei dem - wie in unserer Szene - echohaft Wendungen der ,Vorrednerin' aufgenommen werden.“- Vgl. ferner Besnault/Biтot, Historical legacy (zit. Anm. 5), S. 119f.: „The scene itself, as they sit together and their ,sorrow ... admit[s] society becomes the enactment of a tragic rite. [...] Lamenting women in this play are always three. As a result, they can in turn signify suffering mankind, the three Furies of Greek and Roman mythology who pursue and punish the doers of unavenged crimes, or the three Fates 
Totenklage in II/2 und derjenigen in IV/4 beachtet werden: „When Margaret invokes Heaven it is with a diabolical spirit“", $\left.{ }^{(113}\right)$ und dieser Fremdkörper einer rein christlichen Antiphon bleibt bis zum finalen Abgang von Margaret in IV/4 erhalten: Sie ist und bleibt Richards counterpart; die Ethik von Mt 5,39 wird sie niemals erreichen - und doch ist es gerade ihr weibliches Geschlecht, das sie unüberbrückbar von Richard trennt und zur Leidensgenossin von Königin Elizabeth und der Herzogin von York macht. Auch wenn sie in der Henriade selbst zur Waffe griff: In 'King Richard III ist Margaret auf die kompensatorische - gleichwohl scharfe - Waffe des Fluchs beschränkt, die durch Leiden geschliffen wird. ${ }^{114}$ ) Zwar weigert sie sich, Elizabeth die Kunst wirkungsvollen Verfluchens zu lehren, doch teilt sie ihr mit, nicht sich selbst zum Subjekt ihrer Flüche und Klagen zu machen, sondern das Leid.

Queen Eliz.

My words are dull; O! quicken them with thine! Queen Marg.

Thy woes will make them sharp, and pierce like mine. (V. 123-126, S. 190)

Mit diesem Exit wahrt Margaret nicht nur die ihrem Hoheitsgefühl und ihrer Feindschaft gebührende adlige Distanz, sie wahrt zugleich die in dieser Szene hergestellte Nähe und stößt Elizabeth nicht gänzlich zurück, sondern zeigt ihr die Quelle genuin weiblicher Stärke auf. Die Überlegenheit, mit der Elizabeth Richard in der sich anschließenden Werbungsszene zurückweist, ja vielleicht betrügt, ${ }^{115}$ ) erweckt den Eindruck, dass sich Margarets Stärke tatsächlich auf Elizabeth übertragen hat und sie sich die kalte ,Hilfe zur Selbsthilfe' von ihr zu eigen zu machen vermochte.

Margaret, als eine Art Mischung aus Nemesis und archaisch verfremdeter Marienfigur, hebt den Fluch Englands auf, den Fluch der personifizierten Erbsünde namens Richard, indem sie die Erfüllung ihrer Flüche bewirkt oder, durch das Gebet der Szene IV/4, erwirkt. Anders als Nemesis oder Ate vermag Margaret nämlich nicht aus eigener Kraft zu rächen: Sie bedarf der göttlichen Erhörung und stellt somit eine vermenschlichte, keineswegs allmächtige Nemesis dar. ${ }^{116}$ ) Träte die göttliche Gerechtigkeit im fünften Akt des Dramas nicht unverkennbar durch Richmonds Heer und die Geister der Toten in Erscheinung, verbliebe die

or Parcae who govern human destiny. For a Christian audience, they can also evoke the three Maries at the foot of the Cross. Given the ambivalence of the play and the syncretic spirit at work in the period, they may draw from the audience moral and aesthetic as well as emotional responses."

${ }^{113)}$ Ebenda, S. 118.

$\left.{ }^{114}\right)$ Richard vs. Margaret, das ist Mann vs. Frau, Schwert und Beil vs. Rechtsklage und Fluch, Intellekt und wendige Taktik vs. Magie und steinernes Ritual.

115) Vgl. zu dieser Deutungsoption Clemen, Kommentar (zit. Anm. 5), S. 264.

116) Vgl. ebenda, S. 78. 
magisch-rituelle Macht Margarets auf einer untergründigen, gesellschaftlich subkutanen Ebene.

$$
\begin{aligned}
& \text { Geisterauftritt, ritueller Fluch und Segen (V/3) } \\
& \text { und der Sieg der ,fair ordinance" (V/4) }
\end{aligned}
$$

Let me sit heavy on thy soul to-morrow!

Think how thou stab'dst me in my prime of youth

At Tewskbury: despair, therefore, and die!

[To Richmond.] Be cheerful, Richmond; for the wronged souls

Of butcher'd princes fight in thy behalf:

King Henry's issue, Richmond, comforts thee. (V/3, V. 119-124, S. 234)

Psychologisch ließe sich der serielle Auftritt der elf Geister in V/3 als „Wiederkehr des Verdrängten“, mythologisch als Unbesiegbarkeit einer ,weiblich konnotierten Schicksalslogik' interpretieren. ${ }^{117}$ ) Wichtiger und historisch adäquater scheint jedoch der Hinweis auf das Vorbild des morality play zu sein, dessen dritter Teil den guten Stimmen zum Durchbruch verhilft, wobei die formale Gestaltung durch „Schematismus“, „stereotype Ausdrucksweise“ und eine schlichte „Reihentechnik“" geprägt ist. ${ }^{118}$ ) Darüber hinaus hat die Forschung an das Vorbild der Rachetragödie Senecas erinnert:

Another Senecan feature adapted to the dramatic uses of a hybrid play, they too have a choric and tragic function, as they hammer into Richard's brain formulaic condemnations [...] before they lavish encouragement with identical incantatory force on Richmond: live and flourish!' [...] The curses and blessings of these supernatural apparitions come true at Bosworth. This makes them prophetic instruments of revenge and of divine justice. Again, theatricality vies with pathos and moral implications. For an Elizabethan audience, the final fight was most probably warfare between the forces of Evil and the forces of Good, entrenched on the two sides of the stage, as in earlier Moralities and Interludes, even if the traditional Good and Bad Angels had undergone a tragic metamorphosis. ${ }^{119}$ )

Der aus den Moralities bekannte Kampf zwischen den guten und den bösen Mächten wird in 'King Richard III auch auf lexikalischer Ebene entschieden: zugunsten des durch das Schlüsselwort fair repräsentierten Prinzips. Während Richard durch Alpträume in Nervosität versetzt wird, träumt Richmond ,so fair a dream" (V/3, V. 234, S. 242, Hervorh. v. Ver.), dass er im Vertrauen auf die Hilfe Gottes in die Schlacht gehen kann. Richmond, in der Nachfolge des Friedensfürsten, personifiziert „this fair land's peace“ (V/4, V. 52, S. 254, Hervorh. v. Verf.)

$\left.{ }^{117}\right)$ Shakespeare-Handbuch (zit. Anm. 7), S. 346.

${ }^{118}$ ) Clemen, Kommentar (zit. Anm. 5), S. 292. Vgl. zum „Morality pattern“ außerdem TillYARD, Shakespeare's history plays (zit. Anm. 17), S. 208.

$\left.{ }^{119}\right)$ Besnault/Bitot, Historical legacy (zit. Anm. 5), S. 120. 
und schlichtet den Rosenkrieg durch die ,fair conjunction" der roten und der weißen Rose. (V/4, V. 33, S. 252, Hervorh. v. Verf.)

O! now, let Richmond und Elizabeth, | The true succeeders of each royal house, | By God's fair ordinance conjoin together; And let their heirs - God, if thy will be so -, | Enrich the time to come with smooth-facd'd peace, | With smiling plenty, and fair prosperous days! (V/4, V. 42-47, S. 252-254, Hervorh. v. Verf.)

"God's fair ordinance“ stellt den ordo wieder her; die iniquitas wurde aufgehoben durch das Gesetz göttlicher aequitas, die auch Richmonds militärische Gegner zu versöhnen gewillt sind. Einzig Richard bleibt von der finalen Versöhnung ausgeschlossen; sein letzter Ausruf: „A horse! a horse! my kingdom for a horse“ (V/4, V. 13, S. 250) ist der letzte Einsatz im verlorenen Würfelspiel des Iniquity: Dass Richard sein gesamtes Königreich im Tausch gegen ein einziges Pferd aufs Spiel setzt, zeugt von dem eklatanten Missverhältnis zwischen seinem persönlichen Interesse und dem Wohlergehen seines Staats. ${ }^{120}$ ) Die Balance ist gestört; das Reich ist „out of joint“", 121 ) wenn ein König bereit ist, dieses Reich um den Preis eines Pferds zu verschachern, um für sich eine Überlebenschance zu gewinnen.

So steht der Vers „A horse, a horse, my kingdom for a horse“ für Richards solitäre Position: Er, der Intellektualist, ist zum Reiter ohne Ross geworden, zu einem abstrakten „kingdom“ ohne konkrete Basis, ohne den body politic. ${ }^{122}$ ) „Die extreme Vereinzelung Richards führt dazu, dass er als König seine Integrationsfunktion

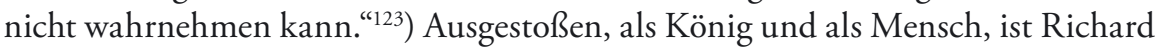
die einzige der dem Untergang geweihten Figuren, die „bezeichnenderweise keinen Bezug auf Margarets Fluch" nimmt. ${ }^{124}$ ) Während jene Opfer Richards, die sich in der Todesstunde an die Prophetin Margaret erinnern, dem reuigen Schächer Golgathas gleichen, erweist sich Richard als ein bis zuletzt verstockter Iniquity, mithin als unerlösbar.

„Fair is foul, and foul is fair" - in 'Macbeth sind es die Hexen, die den Helden mit ihrem Quidproquo des Guten und Bösen, Schönen und Hässlichen verwirren und, dem Handlungsschema der Moralitäten gemäß, auf die Seite des Bösen zie-

$\left.{ }^{120}\right)$ Vgl Clemen, Kommentar (zit. Anm. 5), S. 321: „in diesem symbolischen Notruf wird nun alles, was unter so ungeheurem Aufwand an Blutvergießen und eigenem Einsatz erreicht wurde im Tausch gegen ein Pferd angeboten. So bleibt zum Schluß nur noch die verzweifelte Verteidigung des nackten Lebens im Kampf, und , auf diese Karte` setzt der große Spieler jetzt zum Schluß alles. Es ist wichtig, daß Richard sich selbst am Ende gerade mit einer solchen Metapher des Würfelspiels charakterisiert."

${ }^{121}$ ) Shakespeare, Hamlet (I/5) (zit. Anm. 3), S. 108.

122) Vgl. Tillyard, Shakespeare's history plays (zit. Anm. 17), S. 208: Richard is „the great ulcer of the body politic into which all its impurity is drained and against which all the members of the body politic are united." Ritualtheoretisch betrachtet, müsste Richard demgemäß als „Eliminationsopfer: Sündenbock“ eingestuft werden (DüCKER, Rituale, zit. Anm. 8, S. 140).

$\left.{ }^{123}\right)$ Shakespeare-Handbuch (zit. Anm. 7), S. 345.

${ }^{124}$ ) Clemen, Kommentar (zit. Anm. 5), S. 308. 
hen. In 'King Richard III wird der Protagonist selbst zum equivocator. Nicht auf ihn selbst, doch partiell auf seinen Komplizen Buckingham (sowie am Rande auf die Nebenfiguren Hastings, Rivers, Grey etc.) trifft das von Herbert Weisinger profilierte, "from the myth and ritual pattern" abgeleitete und im morality play vorgebildete Tragödienkonzept zu.

[...] the tragic protagonist, in whom is subsumed the well-being of the people and the welfare of the state, engages in conflict with a representation of darkness and evil; a temporary defeat is inflicted on the tragic protagonist, but after shame and suffering he emerges triumphant as the symbol of the victory of light and good over darkness and evil, a victory sanctified by the covenant of the settling of destinies which reaffirms the well-being of the people and the welfare of the state. In the course of the conflict there comes a point where the protagonist and the antagonist appear to merge into a single challenge against the order of God [...]; and in this moment we are made aware that the real protagonist of tragedy is the order of God against which the tragic hero has rebelled. [...] He commits the foul deed which is potentially in us, he challenges the order of God which we would but dare not, he expiates our sin, and what we had hitherto felt we had been forced to accept we now believe of our free will, namely, that the order of God is just and good..$^{125}$ )

Das hybride Drama 'King Richard III lässt sich - mit Blick auf Richards Opfer-als eine Tragödie interpretieren, deren ,real protagonist [...] the order of God“ ist. Während Richard von Anne als „dreadful minister of hell“ entlarvt wird (I/2, V. 46, S. 20), erscheint Richmond als „God's minister". ${ }^{126}$ Das antithetische Schema der Moralität verstärkt den Fokus des durch Richmond repräsentierten „divine purpose ${ }^{\text {"127) }}$ und lässt das history play an vielen Stellen als heilsgeschichtliches erscheinen. Der ritualhafte Stil vergegenwärtigt eine heilsgeschichtlich grundierte „Anfangsnormativität" der Weltordnung, ${ }^{128}$ ) deren „Invarianz" und „Dauer ${ }^{\text {“129) }}$ durch Figuren der repetitio wie Parallelismus, Anapher, Epipher, Epanalepse, Geminatio, aber auch durch das Strukturprinzip der Symmetrie ${ }^{130}$ ) gestaltet wird.

„God“, der Herr der Heilsgeschichte, soll offenbar nicht nur zu Richmonds Gebet um dauerhaften Frieden „Amen“ sagen, sondern auch zu dieser Gestaltung des Dramenstoffs von ,King Richard III, welche den ordo des Makrokosmos, das aequitas-Prinzip spiegelt. Damit ist nicht gesagt, dass Shakespeare ein „Dramatiker der heilen Welt,“ ein „Verkünder der Schönheit und Wohlgefügtheit des Kos$\operatorname{mos}^{(131)}$ wäre. Seine Dramaturgie des ordo ist gekoppelt an Richard als Spielleiter

\footnotetext{
${ }^{125}$ ) Herbert Weiniger, The Myth and Ritual Approach to Shakespearean Tragedy, in: Centennial Review 1 (1957), S. 142-166, zit. nach: The Myth and Ritual Theory. An Anthology, hrsg. von Robert A. Segal, Oxford; Malden, S. 267-284, hier: S. 275.

$\left.{ }^{126}\right)$ Tillyard, Shakespeare's history plays (zit. Anm. 17), London 1956, S. 204.

${ }^{127}$ ) Besnault/Bitot, Historical legacy (zit. Anm. 5), S. 107.

$\left.{ }^{128}\right)$ Metzler Lexikon Literatur- und Kulturtheorie (zit. Anm. 11), S. 630.

$\left.{ }^{129}\right)$ Ebenda, S. 631.

${ }^{130}$ ) Clemen, Kommentar (zit. Anm. 5), S. 16.

${ }^{131}$ ) Suerbaum, Shakespeares Dramen (zit. Anm. 23), S. 104.
} 
und Margaret als heimliche Regisseurin. Als Meta-Spielleiter lässt Shakespeare iniquity und aequitas interagieren, um diese komplementären Kräfte eines zusehends instabiler werdenden ordo in dynamischer, dennoch stets kontrollierter Balance zu halten. Der ordo wird aufs Spiel gesetzt, und der ordo wird, streng-symmetrisch, restituiert. Aber ohne das Spiel (Goffmansche Theatermetaphorik eingeschlossen) ${ }^{132}$ ) gäbe es keinen ordo für Shakespeare. ${ }^{133}$ )

${ }^{132}$ ) Erving Goffman, Wir alle spielen Theater. Die Selbstdarstellung im Alltag, aus dem Amerikanischen von Peter Weber-Schäfer, 8. Aufl., München und Zürich 2010.

133) Suerbaum, Shakespeares Dramen (zit. Anm. 23), S. 104ff.: „Die intakte Ordnung ist undramatisch. [...] Das theatralische Instrumentarium zur Etablierung der Ordnungen für den Zuschauer [...] ist aufwendig und vielgestaltig: Kostümierung nach Rang und Bedeutung, Aufzüge, Prozessionen, Platznehmen secundum ordinem, Heraushebung der hierarchischen Spitze durch attendants und Befehlsempfänger, eine Dialogführung, die zeigt, wer das Sagen hat und wer nicht. [...] In keinem Stück bleibt die Ordnung so, wie sie anfänglich gezeigt wird. [...] Die Weltordnung dient vor allem als Bezugssystem in einer Welt der Unordnung. Sie ist nur als Norm und als Gegenbild präsent. Sie kann als früher einmal existierend angenommen werden." 
